\title{
phenopype: A phenotyping pipeline for Python
}

\author{
Moritz D. Lürig ${ }^{1,2}$
}

${ }^{1}$ Department of Biology, Lund University, Lund, Sweden

${ }^{2}$ Department of Fish Ecology and Evolution, Eawag, Kastanienbaum, Switzerland

\section{Correspondence}

Moritz D. Lürig

Email: moritz.luerig@gmail.com

Handling Editor: Marta Vidal Garcia

\section{Abstract}

1. Digital images are an intuitive way to capture, store and analyse organismal phenotypes. Many biologists are taking images to collect high-dimensional phenotypic information from specimens to investigate complex ecological, evolutionary and developmental phenomena, such as relationships between trait diversity and ecosystem function, multivariate natural selection or developmental plasticity. As a consequence, images are being collected at ever-increasing rates, but extraction of the contained phenotypic information poses a veritable analytical bottleneck.

2. phenopype is a high-throughput phenotyping pipeline for the programming language Python that aims at alleviating this bottleneck. The package facilitates immediate extraction of high-dimensional phenotypic data from digital images with low levels of background noise and complexity. At the core, phenopype provides functions for rapid signal processing-based image preprocessing and segmentation, data extraction, as well as visualization and data export. This functionality is provided by wrapping low-level computer vision libraries (such as OpenCV) into accessible functions to facilitate scientific image analysis. In addition, phenopype provides a project management ecosystem to streamline data collection and to increase reproducibility.

3. phenopype offers two different workflows that support users during different stages of scientific image analysis. The low-throughput workflow uses regular Python syntax and has greater flexibility at the cost of reproducibility, which is suitable for prototyping during the initial stages of a research project. The high-throughput workflow allows users to specify and store image-specific settings for analysis in human-readable YAML format, and then execute all functions in one step by means of an interactive parser. This approach facilitates rapid program-user interactions during batch processing, and greatly increases scientific reproducibility.

4. Overall, phenopype intends to make the features of powerful but technically involved low-level CV libraries available to biologists with little or no Python coding experience. Therefore, phenopype is aiming to augment, rather than replace the utility of existing Python CV libraries, allowing biologists to focus on rapid and reproducible data collection. Furthermore, image annotations produced by 
phenopype can be used as training data, thus presenting a stepping stone towards the application of deep learning architectures.

\section{KEYWORDS}

automation, computer vision, image analysis, image segmentation, phenomics, phenotype, toolbox, trait measurement

\section{1 | INTRODUCTION}

Digital imaging has become one of the most widespread methods to study phenotypes in ecological and evolutionary research (Lürig et al., 2021; Pennekamp \& Schtickzelle, 2013; Roeder et al., 2012). Produced by a myriad of devices such as digital cameras, scanners and microscopes, high-resolution images capture a variety of internal, external or behavioural characteristics of organisms. In some cases, analysis of digital images replaced manual tasks, for example, when using landmarks instead of manual calipers. Additionally, biologists are using images to draw a more complete picture of organismal phenotypes, for example, by analysing colour (Ezray et al., 2019) and shape (Church et al., 2019) in a continuous fashion, by tracing complex behaviour (Dell et al., 2014; Manoukis \& Collier, 2019) or by capturing microbial diversity (French et al., 2018; Zackrisson et al., 2016). Such data-driven approaches bring the field closer to characterizing the phenome, the phenotype as a whole (Soulé, 1967), and help tracing causal links between phenotypes, genotypes and environmental conditions (Houle et al., 2010). In consequence, digital images are being collected at ever-increasing rates, which has already outpaced our ability to extract and analyse the contained phenotypic information.

Computer vision (CV), the automated extraction and processing of information from digital images, has great potential to reduce image analysis as an analytical bottleneck in ecological and evolutionary research (Høye et al., 2021; Lürig et al., 2021; Weinstein, 2018). CV has already transformed data collection in various fields of research, such as medicine (Gao et al., 2018), remote sensing (Marmanis \& Wegner, 2016) and material sciences (DeCost \& Holm, 2015). In ecological and evolutionary research, however, CV is still only infrequently used to analyse images (Lürig et al., 2021; Weinstein, 2018). Potential hurdles may lie in trade-offs between flexibility and scalability on the one hand, and ease of use on the other hand. For instance, standalone toolboxes with a graphical user interface (GUI) such ImageJ (Schneider et al., 2012) or ilastik (Berg et al., 2019) are often preferred, because they are user friendly and offer a relatively broad range of CV functions. However, batch analysis of larger image datasets is typically more efficient with a high-level programming language, such as Python, which has performant file handling and array computing capabilities, as well as cutting-edge CV libraries, but requires significantly more programming knowledge.

Here I introduce the PYTHON package phenopype, which is a high-throughput image analysis pipeline that allows ecologists and evolutionary biologists to rapidly extract high-dimensional phenotypic data from digital images with low levels of background noise. Specifically, phenopype resolves the aforementioned analytical trade-off by wrapping low level image processing routines into convenient functions with interactive GUI functionality, allowing users to step into an otherwise fully automatic workflow to correct for little differences between images and specimens. phenopype also comes with an efficient file and project management ecosystem to facilitate rapid data collection: all settings for image analysis are stored in human- and machine-readable YAML format along with processed images and results so that the acquired phenotypic information becomes highly reproducible. Overall, phenopype is aiming to augment, rather than replace the utility of existing Python CV libraries. Put differently, phenopype does neither intend to be an exhaustive library of granular image processing functions, like OpenCV or scikit-image, nor a purely GUI-based toolkit like ImageJ, but instead, it is a set of wrappers and convenient management tools that allow biologists to focus on rapid and reproducible data collection.

\section{2 | IMPLEMENTATION}

\section{1 | Capabilities and limitations}

phenopype is intended for the rapid extraction of trait data from digital images with low to moderate amounts of background noise, typically produced under laboratory conditions. Specifically, the specimens to be phenotyped should be photographed against a background with relatively constant colour and brightness (e.g. tray, petri dish, microscope slide, scanner, etc.). This is because phenopype, in its current implementation, uses signal processing for segmentation (see Lürig et al., [2021] for a glossary of CV-terminology); the process of splitting all pixels into foreground (objects of interest) and background (all remaining pixels). The advantages of this segmentation approach are that no GPU or training data are needed, and that all user interactions occur near-instantaneously (depending on image size and CPU performance). Furthermore, users can filter out noise through various preprocessing steps (e.g. using blur kernels, morphology operations or masking), or correct flawed segmentation results with an interactive 'paint-brush'. This makes phenopype especially suitable for tasks that involve the counting of specimens, shape measurements (up to 41 shape features), the extraction of colour intensities and texture from different colour channels (up to 120 texture features per channel), and movement recognition. For an overview of phenopype's capabilities, refer to Section 4 or check the package homepage (www.phenopype.org). 


\section{2 | Package dependencies}

phenopype (2.1.0) is implemented in Python 3.7. It relies on the Python Standard Library and several external dependencies, which are also downloaded during package installation (Figure 1). Since images are represented as multidimensional arrays in Python, the numpy library (Harris et al., 2020) is needed for basic array handling and computing capabilities. The OpenCV library (Bradski, 2000), implemented through the opencv-python package, serves as the main backend for most image processing and analysis functionality (contained in the core modules), and for basic image I/O utility functions (Figure 1A). Furthermore, phenopype uses the pyradiomics library (van Griethuysen et al., 2017) for the extraction of texture features. phenopype serves as a wrapper for opencv-python and pyradiomics, whose CV functions operate on a low level and thus require some basic checks and preparation steps (Figure 1B). The ruamel.yaml package is used to load and parse user modifications, and watchdog, a package that detects changes to files, is used to provide an interactive user experience while modifying YAML files.

\section{3 | Package API}

The complete API (application programming interface) is available in the online documentation (www.phenopype.org), but a brief overview is given here:
1. The project management class (Project) and its methods (e.g. add_files, add_config, collect_results, etc.) are intended to systematically select images on the hard drive and load them into an organized folder structure.

2. Various utility functions for loading, inspecting and saving images (e.g. load_image, show_image, save_image, etc.).

3. The five image analysis core modules (preprocessing, segmentation, measurement, visualization and export), with low-level CV tools from the opencv-python library wrapped into convenient functions (see Figure 1B for a schematic). Some of these functions open a graphical user interface (GUI).

4. The Pype class is an interactive parser, translating user instructions stored in YAML into Python code (see Figure $3 \mathrm{C}$ for details).

\section{3 | GETTING STARTED WITH PHENOPYPE}

\section{1 | Installation and setup}

To establish a Python 3.7 environment and to ensure the proper installation of all dependencies, the use of a Python environment manager is highly recommended (e.g. Anaconda). After setting up an environment, phenopype can be installed from the Python Package Index (PyPI; https://pypi.org/project/phenopype/) with pip install phenopype. Lastly, a text editor capable of opening YAML files (without locking them) needs to be installed and selected as the default program.

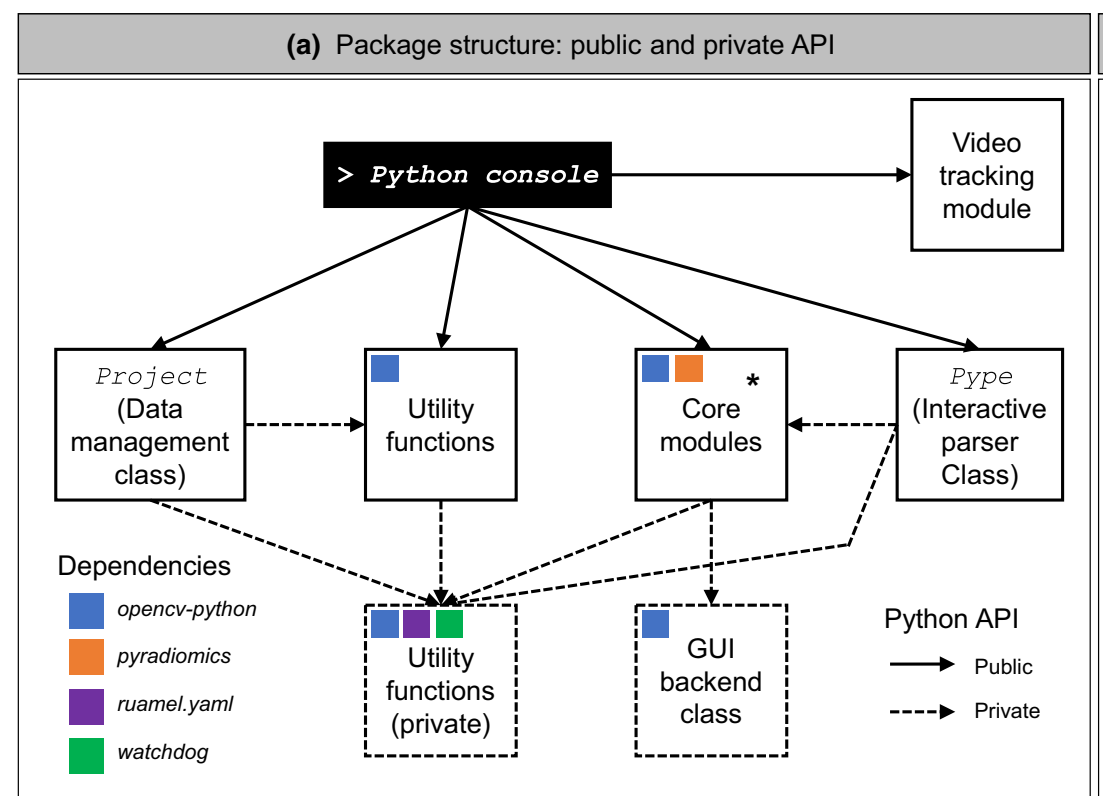

(b) Internals of a phenopype function (schematic)

* phenopype.core.segmentation.thresholdl image, method, value, blocksize, constant, channel, mask)

Check image datatype and dimension:

a) If muldichannel, select a specific channel (provided by channel [e.g. gray or RGB])

b) If checks fail, abort

Apply a flavor of opencv-python's thresholding function (method = "binary", "otsu", or "adaptive"):

a) cv2.threshold("binary", value)

b) cv2.threshold("otsu")

c) cv2.adaptiveThreshold("adaptive", blocksize, constant)

If provided, combine binary mask annotations with thresholded image to include or exclude areas

$\downarrow$

Return thresholded image as binary mask (Fig. 2A-iii)

FIGURE 1 Schematic of the package design. (a) The public API provides access to project management and utility functions (e.g. for loading and saving images), the core modules, which contain all image processing functions, and the Pype class, which is an interactive parser used in the high-throughput workflow (see Section 3.2.2). (b) phenopype does not supply its own technical basis for image analysis, but instead 'wraps' existing low-level computer vision functions into convenient functions that perform all necessary checks and conversions. Here, a schematic for such a wrapper function (threshold function from the segmentation module) is given to exemplify how external libraries are integrated into phenopype 


\subsection{Working with phenopype}

In phenopype, users can choose between 'low-throughput' and 'highthroughput' workflows, each of which accommodate the different stages in the process of scientific image analysis. Early during an ongoing experiment or a survey, when images are first evaluated on the computer screen, scientific image analysis can be a highly iterative process that may require frequent user input to preprocess and segment images, and then evaluate the obtained results. This may be faster and more intuitive with the low-throughput workflow, which allows inspection of the modified arrays and annotations in Python. Later, when the best functions and appropriate settings are found and rapid data collection has priority, image analysis should be seamless and with minimal user input for maximal throughput, but also with maximal scientific reproducibility, which is ensured with the high-throughput workflow.

\subsection{1 | Low-throughput workflow}

In the low-throughput workflow, images are loaded as arrays using the utility functions, and then processed and analysed using CV functions from the five core modules are called in Python (Figure 2B). This allows users to interact with arrays directly, which is providing a good sense of which function is doing what and likely a good starting point for people with no prior exposure to the concepts of CV. However, this also means that settings need to be modified for each image separately, which makes this workflow inconvenient for larger datasets.

\subsection{2 | High-throughput workflow}

Here, instead of coding each function for each image, the entire workflow is stored in human readable YAML format and can be called by a special interactive parser class (Pype class; Figures $2 \mathrm{C}$ and 3). This dramatically reduces user input, and is thus suitable for the processing of larger datasets (100s-1,000s of images) by using the Pype class in a simple for loop (Figure 3C). To further support batch processing, phenopype provides convenient project management functions to store and organize raw image data, YAML configurations, and results in a directory tree (Project class; Figure 3).

\subsection{3 | Video analysis}

phenopype also includes a video analysis module which allows segmentation of video frames using foreground-background subtraction algorithms. Such algorithms first determine what constitutes the background by detecting static pixels over a range of frames, and then determine foreground by identifying pixels of objects that deviate from the background model through movement. The video module, although fully functional and with complete documentation, is currently only weakly integrated into the different phenopype workflows, and is listed here only for completeness.

\section{4 | FEATURE DEMONSTRATION}

phenopype can be used to extract a wide array of organismal traits, particularly those related to external shape and texture, and it has already proven its effectiveness by producing large amounts of phenotypic data for two peer-reviewed publications (Lürig et al., 2019; Lürig \& Matthews, 2021). Here I demonstrate some of phenopype's key features and provide some examples of different types of trait data that can be extracted. The following paragraphs correspond to the panels in Figure 4. More extensive examples with code can be found on the package homepage (www.pheno pype.org):

(a) Counting snails: Counting small organisms in the laboratory is a task that has good potential to be solved automatically with CV. Here, phenopype was used to count freshwater snails Potamopyrgus antipodarum, and measure their body size, shape and pigmentation. This was achieved through consecutive use of threshold (to segment black snails from white background), and watershed algorithms (to separate snails that are touching each other). Small faecal pellets are filtered from the detected foreground (the green contours) using blur kernels and a size cut-off. Corrections for the gradient of brightness in the image are conducted by comparing each specimen to the background within its bounding box.

(b) Stickleback landmarks and armor plating: Functional morphology of organisms is often measured by placing landmarks at specific points, but variation in continuous phenotypic traits like shape or area are difficult to quantify manually because they are too complex or have no underlying assumption of homology. In this example, the traits of interest are contained in 22 landmarks that were set with phenopype across the anterior half of a threespine stickleback Gasterosteus aculeatus, which was stained with alizarin red. In addition, the number, area and extension of armour plating was measured as a continuous trait: first, a mask was set around the posterior region that contains the plates, then the red colour channel of the image, which contained the highest signal-to-noise ratio, was thresholded.

(c) Venation patterns in damselfly wings: Here, phenopype was used to measure the spacing, angles and connectivities within the wing tissue of an adult damselfly Ischnura elegans. Because the venation within the wing has an extremely high signal-to-noise ratio, a single thresholding algorithm could be used without further preprocessing. The full hierarchy of foreground contours was resolved: the outer perimeter of the whole wing (in green) and all domains within the wing (in red).

(d) Isopod movement in response to predation: In this example, the goal was to track the movement of freshwater isopods Asellus aquaticus in response to presence, absence and activity of a predator $G$. aculeatus. The output of the phenopype's video tracking module 


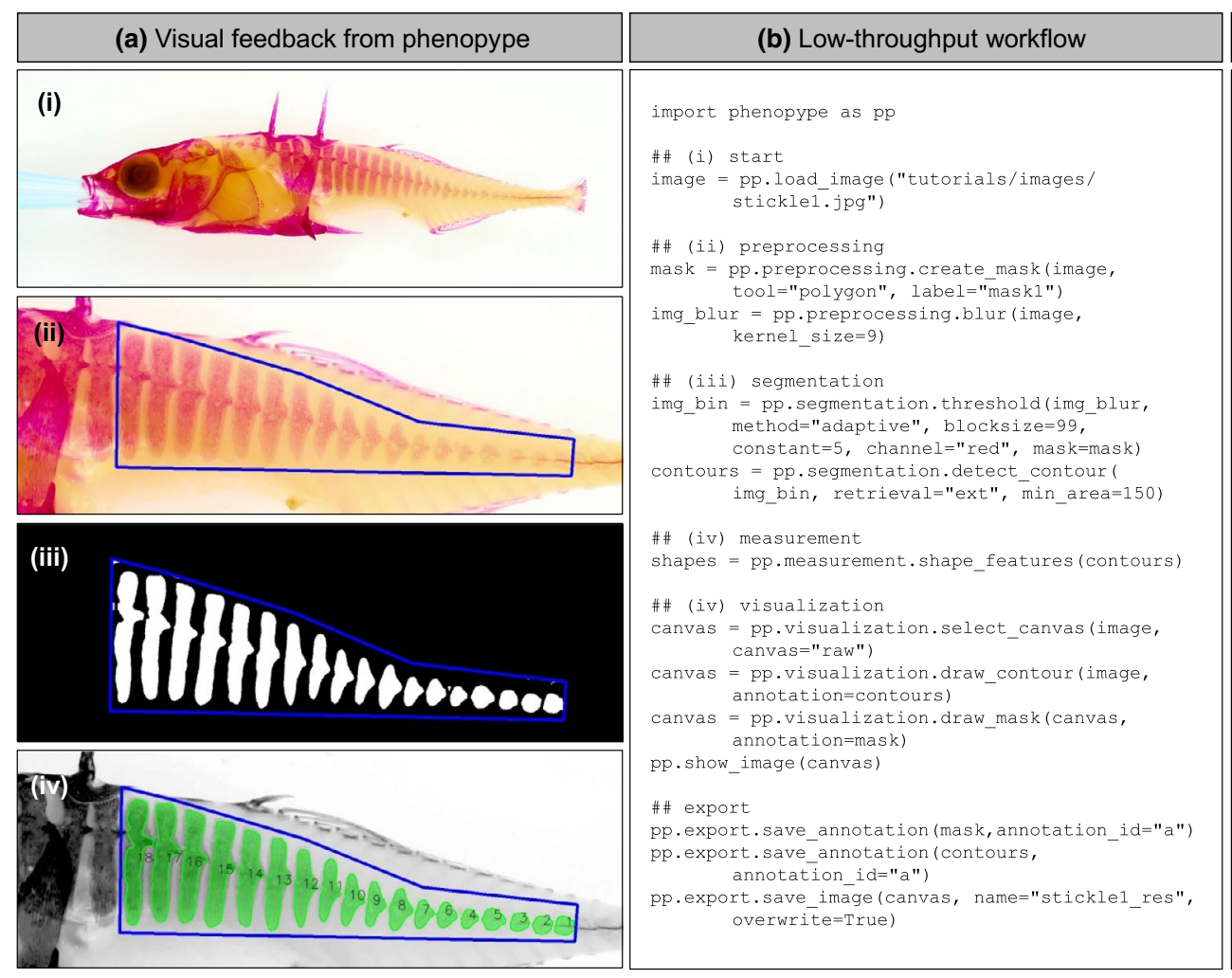

(c) High-throughput workflow

import phenopype as pp
pp.Pype ("tutorials/images/
stickle1.jpg", name="demo_v1",
config="config.yam1")

Content of config.yaml:

processing_steps:

- preprocessing:

preprocessing:

tool: polygon

- blur:

kernel_size: 9

- segmentation:

threshold:

method: adaptive

blocksize: 99

constant: 5

channel: red

- detect_contours:

retrieval: ext

min_area: 150

measurement:

- shape_features

visualization:

- select_canvas:

canvas: raw

draw contour

- draw mask

- export

- save_annotations

save_canvas:

overwrite: true

FIGURE 2 An exemplified computer vision task in phenopype to measure the number, area and shape of bony armour plating in a stained specimen of threespine stickleback Gasterosteus aculeatus. Visual feedback (a) from low-throughput (b) and high-throughput workflow (c), which use the same computer functions, but differ in the way these functions are called by the user: while in the low-throughput workflow, all functions have to be explicitly coded in Python, the high-throughput routine parses the functions from human readable YAML files to facilitate rapid user interaction and increase reproducibility (see Section 3.2 for details on both workflows).

is a time series of coordinates that correspond to the trajectories of predator and prey. In addition, the foreground-background subtraction algorithm produces a binary segmentation mask for every frame. This allows the detection of pigmentation and body size of every isopod, and thus the sequence in which the fish consumed differently pigmented and sized specimens.

(e) Phytoplankton cell shape and fluorescence: A plate reader produced bright-field and fluorescence emission images of freshwater phytoplankton communities (shown here is Pediastrum sp.). Here the goal was to measure the shape (cell morphology) and texture (fluorescence intensity) of cells. This was done in a two-step process: first, the brightfield image was thresholded to detect the boundary contours of the cell. Then, the contours were used as a stencil on images of different fluorescence channels that capture variation in three pigments (chlorophyll, phycoerythrin and phycocyanin), measuring intensities within the contour of the cell.

(f) Automatic reference card detection: Even in highly standardized laboratory environments, there are often slight variations in camera exposure and zoom that may affect the outcome of measurements and extracted traits. To automate the time-consuming task of correcting for size ratio and brightness variation, phenopype provides a suite of performant image registration algorithms (Tareen \& Saleem, 2018) to detect reference cards within images, and automatically correct size and colour histograms of that image. This can be done on a project-wide scale, where first a template is created, and then detected in every following image.

\section{5 | DISCUSSION AND OUTLOOK}

With phenopype, I have presented a novel approach for rapid, reproducible and user-friendly extraction of phenotypic data from digital images. Its main innovation lies in making low-level CV functions more accessible through code wrapping and markup language that is both human and machine readable: using YAML configuration files instead of Python code, users can assemble functions in manifold ways to address the specifics of their study organism, while maintaining cross-platform compatibility and achieving complete reproducibility. Together with a simple GUI and a convenient project management ecosystem, this approach provides a seamless workflow for larger image datasets. Initially, users may take some time to learn and understand the approach. Here the low-throughput workflow can serve as an easy introduction to CV in Python. In the long run, the initial time investment will pay off, as tedious and repetitive tasks are eliminated with the high-throughput workflow, which should save a lot of time during image analysis and project organization. 
(a) Automatically organize images into directory tree
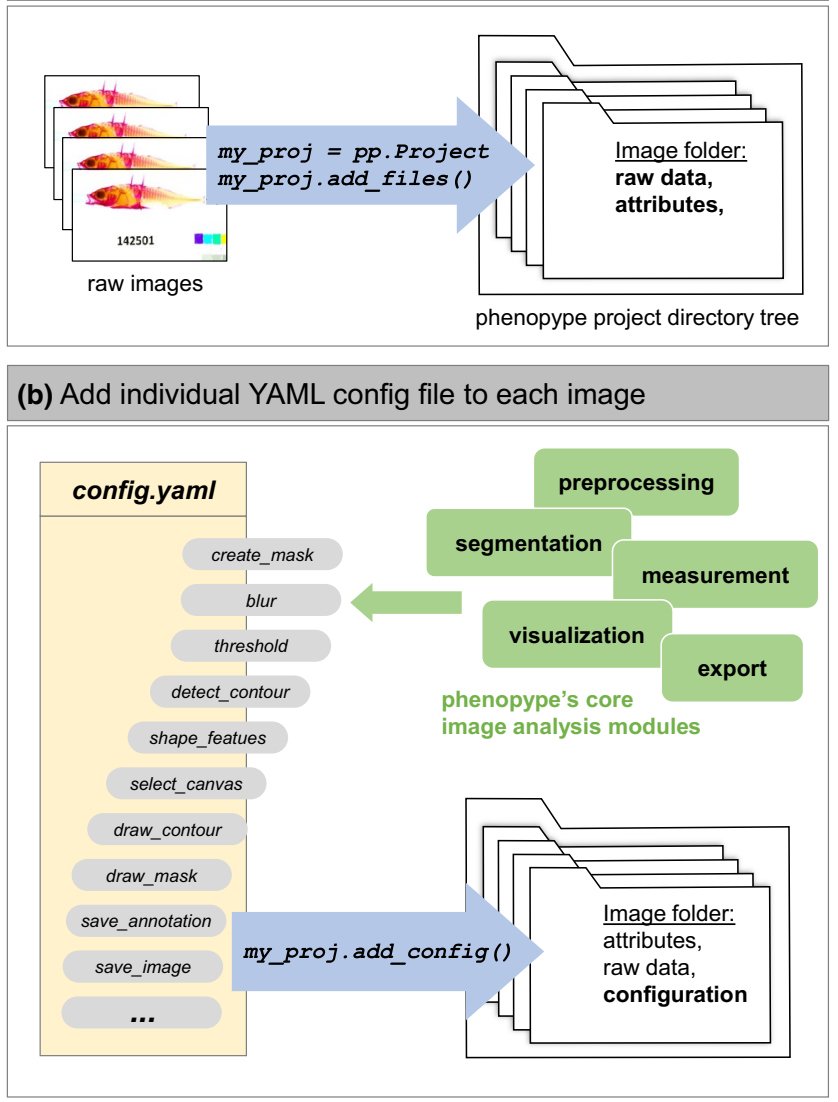

(c) Apply the Pype class and all its steps (i-iii) to each image for folder in my_proj.dirpaths:

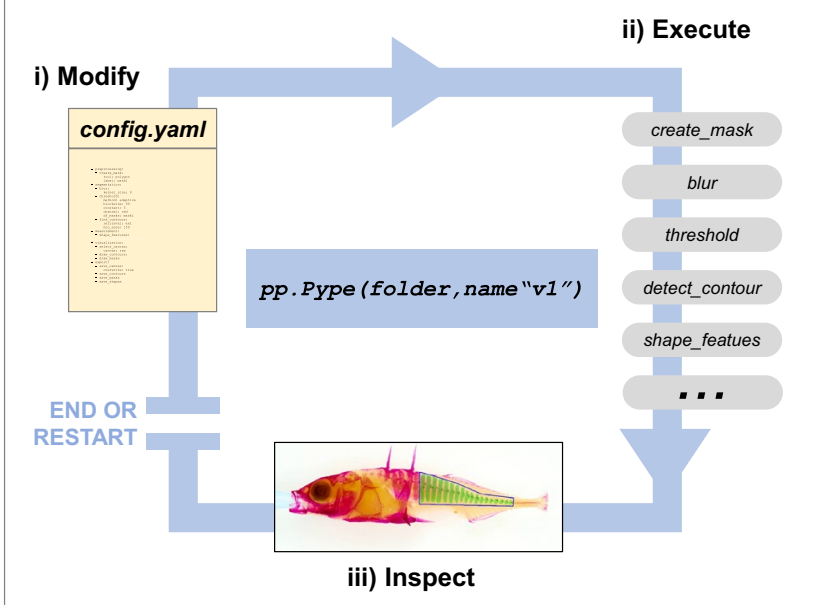

(d) Bundle results; run again; try different configurations

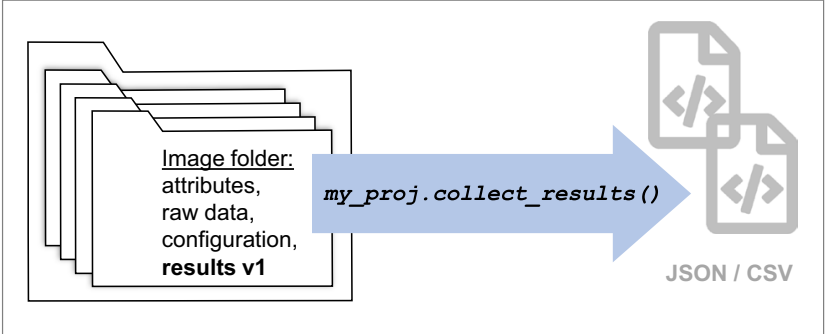

FIGURE 3 Flow chart of the high-throughput workflow in phenopype. (a) First a Project class is initialized, yielding the class object 'my_proj'. Then, using the class method add_files, a directory tree is be created where all relevant files are stored. (b) Next a configuration file in YAML format is created and stored within each folder of the phenopype directory tree using class method add_config. Users can choose between one of several configuration templates for different cases (e.g. counting landmarking, colour scoring or shape measurement, and more). (c) The Pype class in a for loop will trigger a series of events for each image directory provided by the loop generator: (i) open the contained yaml configuration with the default OS text editor, (ii) parse and execute the contained functions from top to bottom, (iii) open a GUI window and show the processed image. Once the Pype class has finished executing all functions from the configuration file, users can decide to either modify the opened configuration file (e.g. either change function parameters or add new functions), which will trigger to run the Pype class again, or to close the GUI window, which will terminate the Pype class instance and save all results to the folder. By including the Pype class in a simple Python for-loop with all project folders, users can continue with this procedure throughout the entire project dataset, eliminating the need to manually open, close or save any of the images. (d) When all images have been analysed, the obtained results are obtained from each project folder with the class method collect_results, and store them in a new folder in the project root directory for further analysis in their favourite statistical environment

phenopype provides $\mathrm{CV}$ functions that are based on signal processing, which works instantaneously (i.e. it does not require any training), has lower technical hurdles (no GPU required) and is less complex to implement, but also prone to higher levels of background noise and convolution. Therefore, the package is intended as a pipeline for scientific photography dedicated to phenotyping, where by default great care is taken to minimize levels of background noise. While for this purpose machine learning is typically not needed to achieve good segmentation, it moreover is often not even possible, because training data, sufficient time, or GPU hardware appropriate for deep learning are lacking (Lürig et al., 2021). For cases without these constraints, with very large datasets, or with high levels of noise, recent innovative Python machine learning toolkits like ml-morph (Porto \& Voje, 2020) or sashimi (Schwartz \& Alfaro, 2021) are recommended alternatives.

Regardless of potential constraints or scope of the research, phenopype may serve as a stepping stone towards such machine learning approaches, because it produces foreground masks as a 'byproduct' and thus provides labelled training datasets, which are an increasing bottleneck for deep learning approaches (Sun et al., 2017). In the long term and with the help and feedback of the CV community, a deep learning workflow could be integrated to phenopype: on the one hand, Python, specifically the OpenCV library that phenopype is using as a backend, already comes with a suite of state-of-the-art deep learning implementations, which are frequently updated with the most cutting edge algorithms (such as Mask-RCNN; Abdulla, 2017). On the other hand, phenopype's modular architecture facilitates the inclusion of current and future advances within the highly dynamic field of scientific CV. 

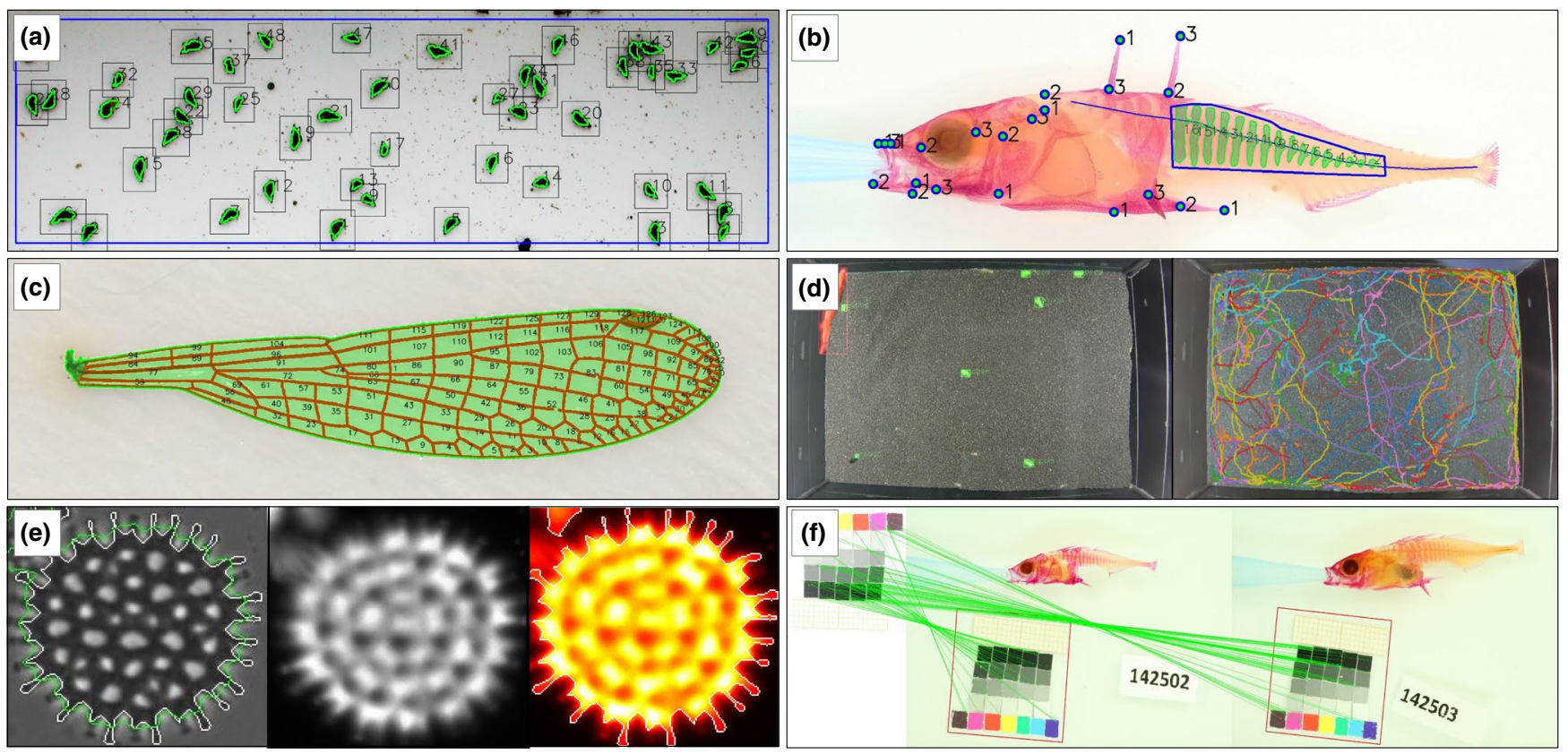

FIGURE 4 Demonstration of major phenopype features (the panels correspond to the paragraphs in Section 4). (a) Counting and measuring freshwater snails (Potamopyrgus antipodarum; image by Jukka Jokela). (b) Landmarking and armour plate measurement in threespine stickleback (Gasterosteus aculeatus; image by Dominique Stalder). (c) Quantifying venation patterns in damselfly wings (Ischnura elegans; image by Masahito Tsuboi). (d) Tracking predator prey interactions between stickleback (G. aculeatus) and freshwater isopods (Asellus aquaticus; video shot by Kim Kaltenbach). (e) Extracting shape and fluorescence traits from phytoplankton cells (Pediastrum sp.; image by Irene Gallego). (f) Automatic detection of reference cards in images using phenopype (image by Dominique Stalder)

\section{ACKNOWLEDGEMENTS}

I conceived phenopype in its current form during a laboratory retreat in Vna (Graubünden, Switzerland) that was organized and funded by Jukka Jokela. Its implementation was made possible by Blake Matthews and the Eawag directorate (Discretionary Funding Grant No. 5221.00492.013.11). Additional funding came from the Swiss National Science Foundation through an Early Postdoc. Mobility Fellowship (Grant No. P2EZP3_191804) and from the European Union's Horizon 2020 research and innovation programme through a Marie Skłodowska-Curie IF (Grant No. 898932). I thank Kim Kaltenbach for being a patient alpha tester, and Cam Hudson, Ryan Greenway, Andres Grolimund, Nare Ngoepe, Anja Merz and Irene Gallego for being helpful beta testers. I would also express my sincere gratitude to Arthur Porto and Seth Donoughe whose comprehensive review for the pyOpenSci consortium greatly improved the presentation and documentation of the package. Two anonymous reviewers provided very constructive feedback during review of the manuscript. The stickleback image for phenopype's logo was taken by Angelina Arquint. Finally, this package may not have come into existence without Matt McGee, who encouraged me to learn Python and use it for computer vision.

\section{CONFLICT OF INTEREST}

I declare the absence of any scientific, commercial or financial relationships that could be construed as a potential conflict of interest.

\section{PEER REVIEW}

The peer review history for this article is available at https://publo ns.com/publon/10.1111/2041-210X.13771.

\section{DATA AVAILABILITY STATEMENT}

A snapshot of phenopype $\mathrm{v}$ 2.1.0 (including all electronic supplementary materials), which this publication is based on, can be found on Zenodo (Lürig, 2021). The latest release, installation instructions, the full documentation and API reference, as well as tutorials and vignettes can be found on the project homepage (www.phenopype. org).

\section{ORCID}

Moritz D. Lürig (D) https://orcid.org/0000-0002-8175-6234

\section{REFERENCES}

Abdulla, W. (2017). Mask r-cnn for object detection and instance segmentation on keras and tensorflow. Retrieved from https://github.com/ matterport/Mask_RCNN

Berg, S., Kutra, D., Kroeger, T., Straehle, C. N., Kausler, B. X., Haubold, C., Schiegg, M., Ales, J., Beier, T., Rudy, M., Eren, K., Cervantes, J. I., Xu, B., Beuttenmueller, F., Wolny, A., Zhang, C., Koethe, U., Hamprecht, F. A., \& Kreshuk, A. (2019). ilastik: Interactive machine learning for (bio)image analysis. Nature Methods, 16(12), 1226-1232. https:// doi.org/10.1038/s41592-019-0582-9

Bradski, G. (2000). The opencv library. Doctor Dobbs Journal, 25(11), 120-126.

Church, S. H., Donoughe, S., de Medeiros, B. A. S., \& Extavour, C. G. (2019). Insect egg size and shape evolve with ecology but not 
developmental rate [Review of Insect egg size and shape evolve with ecology but not developmental rate]. Nature, 571(7763), 5862. https://doi.org/10.1038/s41586-019-1302-4

DeCost, B. L., \& Holm, E. A. (2015). A computer vision approach for automated analysis and classification of microstructural image data. Computational Materials Science, 110, 126-133. https://doi. org/10.1016/j.commatsci.2015.08.011

Dell, A. I., Bender, J. A., Branson, K., Couzin, I. D., de Polavieja, G. G., Noldus, L. P. J. J., Pérez-Escudero, A., Perona, P., Straw, A. D., Wikelski, M., \& Brose, U. (2014). Automated image-based tracking and its application in ecology. Trends in Ecology \& Evolution, 29(7), 417-428. https://doi.org/10.1016/j.tree.2014.05.004

Ezray, B. D., Wham, D. C., Hill, C. E., \& Hines, H. M. (2019). Unsupervised machine learning reveals mimicry complexes in bumblebees occur along a perceptual continuum. Proceedings of the Royal Society $B$ : Biological Sciences, 286(1910), 20191501. https://doi.org/10.1098/ rspb.2019.1501

French, S., Coutts, B. E., \& Brown, E. D. (2018). Open-source highthroughput phenomics of bacterial promoter-reporter strains. Cell Systems, 7(3), 339-346.e3. https://doi.org/10.1016/j. cels.2018.07.004

Gao, J., Yang, Y., Lin, P., \& Park, D. S. (2018). Computer vision in healthcare applications. Journal of Healthcare Engineering, 2018, 5157020. https://doi.org/10.1155/2018/5157020

Harris, C. R., Millman, K. J., van der Walt, S. J., Gommers, R., Virtanen, P., Cournapeau, D., Wieser, E., Taylor, J., Berg, S., Smith, N. J., Kern R., Picus, M., Hoyer, S., van Kerkwijk, M. H., Brett, M., Haldane, A., Del Río, J. F., Wiebe, M., Peterson, P., ... Oliphant, T. E. (2020). Array programming with NumPy. Nature, 585(7825), 357-362. https:// doi.org/10.1038/s41586-020-2649-2

Houle, D., Govindaraju, D. R., \& Omholt, S. (2010). Phenomics: The next challenge. Nature Reviews Genetics, 11(12), 855-866. https://doi. org/10.1038/nrg2897

Høye, T. T., Ärje, J., Bjerge, K., Hansen, O. L. P., losifidis, A., Leese, F., Mann, H. M. R., Meissner, K., Melvad, C., \& Raitoharju, J. (2021). Deep learning and computer vision will transform entomology. Proceedings of the National Academy of Sciences of the United States of America, 118(2), e2002545117. https://doi.org/10.1073/ pnas. 2002545117

Lürig, M. D. (2021). Data from: phenopype: A phenotyping pipeline for Python. Zenodo, https://doi.org/10.5281/zenodo.5645001

Lürig, M. D., Best, R. J., Svitok, M., Jokela, J., \& Matthews, B. (2019). The role of plasticity in the evolution of cryptic pigmentation in a freshwater isopod. The Journal of Animal Ecology, 88(4), 612-623. https:// doi.org/10.1111/1365-2656.12950

Lürig, M. D., Donoughe, S., Svensson, E. I., Porto, A., \& Tsuboi, M. (2021). Computer vision, machine learning, and the promise of phenomics in ecology and evolutionary biology. Frontiers in Ecology and Evolution, 9, 148. https://doi.org/10.3389/fevo.2021.642774

Lürig, M. D., \& Matthews, B. (2021). Dietary-based developmental plasticity affects juvenile survival in an aquatic detritivore. Proceedings of the Royal Society B: Biological Sciences, 288(1945), 20203136. https://doi.org/10.1098/rspb.2020.3136

Manoukis, N. C., \& Collier, T. C. (2019). Computer vision to enhance behavioral research on insects. Annals of the Entomological Society of America, 112(3), 227-235. https://doi.org/10.1093/aesa/say062

Marmanis, D., \& Wegner, J. D. (2016). Semantic segmentation of aerial images with an ensemble of CNNs. Annals of the Photogrammetry,
Remote Sensing and Spatial Information Sciences. Retrieved from https://www.isprs-ann-photogramm-remote-sens-spatial-inf-sci. net/III-3/473/2016/isprs-annals-III-3-473-2016.pdf

Pennekamp, F., \& Schtickzelle, N. (2013). Implementing image analysis in laboratory-based experimental systems for ecology and evolution: A hands-on guide. Methods in Ecology and Evolution/British Ecological Society, 4(5), 483-492. https://doi.org/10.1111/2041-210X.12036

Porto, A., \& Voje, K. L. (2020). ML-morph: A fast, accurate and general approach for automated detection and landmarking of biological structures in images. Methods in Ecology and Evolution, 11(4), 500512. https://doi.org/10.1111/2041-210X.13373

Roeder, A. H. K., Cunha, A., Burl, M. C., \& Meyerowitz, E. M. (2012). A computational image analysis glossary for biologists. Development, 139(17), 3071-3080. https://doi.org/10.1242/dev.076414

Schneider, C. A., Rasband, W. S., \& Eliceiri, K. W. (2012). NIH Image to ImageJ: 25 years of image analysis. Nature Methods, 9(7), 671-675. https://doi.org/10.1038/nmeth.2089

Schwartz, S. T., \& Alfaro, M. E. (2021). Sashimi: A toolkit for facilitating high-throughput organismal image segmentation using deep learning. Methods in Ecology and Evolution/British Ecological Society, 2041210X.13712. https://doi.org/10.1111/2041-210x.13712

Soulé, M. (1967). Phenetics of natural populations I. Phenetic relationships of insular populations of the side-blotched lizard. Evolution: International Journal of Organic Evolution, 21(3), 584-591. https:// doi.org/10.1111/j.1558-5646.1967.tb03413.x

Sun, C., Shrivastava, A., Singh, S., \& Gupta, A. (2017). Revisiting unreasonable effectiveness of data in deep learning era. arXiv [cs.CV] arXiv. http://arxiv.org/abs/1707.02968

Tareen, S. A. K., \& Saleem, Z. (2018). A comparative analysis of SIFT, SURF, KAZE, AKAZE, ORB, and BRISK. ResearchGate. 2018 International Conference on Computing, Mathematics and Engineering Technologies - iCoMET 2018, March 4. https://doi. org/10.1109/ICOMET.2018.8346440

van Griethuysen, J. J. M., Fedorov, A., Parmar, C., Hosny, A., Aucoin, N., Narayan, V., Beets-Tan, R. G. H., Fillion-Robin, J.-C., Pieper, S., \& Aerts, H. J. W. L. (2017). Computational radiomics system to decode the radiographic phenotype. Cancer Research, 77(21), e104-e107. https://doi.org/10.1158/0008-5472.CAN-17-0339

Weinstein, B. G. (2018). A computer vision for animal ecology. The Journal of Animal Ecology, 87(3), 533-545. https://doi. org/10.1111/1365-2656.12780

Zackrisson, M., Hallin, J., Ottosson, L.-G., Dahl, P., Fernandez-Parada, E., Ländström, E., Fernandez-Ricaud, L., Kaferle, P., Skyman, A., Stenberg, S., Omholt, S., Petrovič, U., Warringer, J., \& Blomberg, A. (2016). Scan-o-matic: High-resolution microbial phenomics at a massive scale. G3, 6(9), 3003-3014. https://doi.org/10.1534/ g3.116.032342

How to cite this article: Lürig, M. D. (2022). phenopype: A phenotyping pipeline for Python. Methods in Ecology and Evolution, 13, 569-576. https://doi.org/10.1111/2041210X.13771 